\title{
WHEN NONLINEAR DIFFERENTIAL EQUATIONS ARE EQUIVALENT TO LINEAR DIFFERENTIAL EQUATIONS*
}

\author{
SUKEYUKI KUMEI $\dagger$ AND GEORGE W. BLUMAN $\dagger$
}

\begin{abstract}
A necessary and sufficient condition is established for the existence of a 1-1 transformation of a system of nonlinear differential equations to a system of linear equations. The obtained theorems enable one to construct such transformations from the invariance groups of differential equations. The hodograph transformation, the Legendre transformation and Lie's transformation of the Monge-Ampère equation are shown to be special cases. Noninvertible transformations are also considered. Examples include Burgers' equation, a nonlinear diffusion equation and the Liouville equation.
\end{abstract}

Introduction. In this work, we study transformations mapping nonlinear differential equations to linear differential equations in a 1-1 manner. Based upon the group analysis of differential equations, we obtain necessary and sufficient conditions for the existence of such transformations. The established theorems not only allow us to determine the existence of the transformations but also enable us to actually construct these transformations from invariance groups of the nonlinear equations.

In the following analysis, two types of transformations are considered: (1) the invariance groups of differential equations; and (2) the mappings which transform nonlinear differential equations to linear differential equations. Theorems will be proved based upon the following observations. Clearly if there exists a 1-1 mapping between any two differential equations it must inject properties of one equation into the other, including their invariance properties. For this reason the often ignored fact that any linear differential equation admits an invariance group related to the superposition principle becomes very significant. The generator of this particular group depends upon an arbitrary solution of the linear equation. It follows then that any nonlinear equation transformable to a linear equation by a 1-1 mapping must admit an invariance group whose generator depends upon an arbitrary solution of some linear differential equation.

The idea of comparing invariance groups of differential equations in the search of mappings connecting the equations was first used by Bluman in his study of Burgers' equation [1] and it was applied to the study of the mappings of one-dimensional linear parabolic equations to the heat equation [2].

In the first section of this paper some basic properties of various transformations are summarized. The theorems are presented in the second section. The examples in the third section include the hodograph transformation, the Legendre transformation, Lie's transformation of the Monge-Ampère equation and the equation $\left(u_{x}\right)^{\alpha} u_{x x}-u_{y y}=$ 0 . In the last section we examine properties of noninvertible mappings of Burgers' equation, the diffusion equation $\left(u^{-2} u_{x}\right)_{x}-u_{y}=0$ and the Liouville equation $u_{x y}=e^{u}$, respectively, into linear equations.

1. Transformations of invariance groups. We consider a 1-1 mapping $T$ of a vector space $w$ to a vector space $W$. In this section we are interested in how a generator of a Lie group defined in one space is mapped into the other space by $T$.

Throughout the paper we adopt the customary summation rule for repeated indices. Italic indices are summed from 1 to $M$, and Greek indices from 1 to $N$.

* Received by the editors March 26, 1981.

$\dagger$ Department of Mathematics and Institute of Applied Mathematics and Statistics, University of British Columbia, Vancouver, British Columbia, Canada V6T 1 Y4. 
1.1. Invariance groups [3]-[6]. We consider an infinite dimensional vector space $w$ with coordinates

$$
\omega=(x, z, \underset{1}{z}, \underset{2}{z}, \cdots, \underset{n}{z}, \cdots)
$$

where $x=\left(x_{1}, x_{2}, \cdots, x_{M}\right), z=\left(z^{1}, z^{2}, \cdots, z^{N}\right)$. The coordinates of $z$ consist of $z_{i_{1} i_{2} \cdots i_{n}}^{\mu} \quad$ with $\mu=1,2, \cdots, N$ and $i_{k}=1,2, \cdots, M$. For instance, $z=$ $\left(z_{11}^{1}, \cdots, z_{M M}^{1}, \cdots, z_{11}^{N}, \cdots, z_{M M}^{N}\right)$. We adopt a notation $w^{(n)}$ for the vector space with coordinates

$$
\omega^{(n)}=(x, z, \underset{1}{z}, \cdots, \underset{n}{z})
$$

In the later analysis of differential equations, we associate with $z^{\mu}$ a function $u^{\mu}(x)$ and with $z_{i j \cdots n}^{\mu}$ a derivative $u_{i j \cdots n}^{\mu}(x)=\partial_{x_{i}} \partial_{x_{j}} \cdots \partial_{x_{n}} u^{\mu}(x)$.

Let $\gamma$ be the space of functions $w^{(k)} \rightarrow R, k$ finite, analytic in a given domain $D(w)$ in $w$. We consider an operator of the form

$$
l=\xi^{i} \partial_{x_{i}}+\zeta^{\mu} \partial_{z^{\mu}}+\zeta_{i}^{\mu} \partial_{z_{i}^{\mu}}+\cdots+\zeta_{i_{1} i_{2} \cdots i_{k}}^{\mu} \partial_{i_{1} i_{2} \cdots i_{k}}^{\mu}+\cdots,
$$

$\xi^{i} \in \gamma, \zeta^{\mu} \in \gamma$ and

$$
\zeta_{i \cdots j k}^{\mu}=D_{x_{k}} \zeta_{i \cdots j}^{\mu}-z_{i \cdots j m}^{\mu} D_{x_{k}} \xi^{m}
$$

where

$$
D_{x_{k}}=\partial_{x_{k}}+z_{k}^{\mu} \partial_{z^{\mu}}+z_{i k}^{\mu} \partial_{z_{i}^{\mu}}+z_{i j k}^{\mu} \partial_{z_{i j}^{\mu}}+\cdots ;
$$

$l$ is a linear operator $\gamma \rightarrow \gamma$. The linear space of operators $l$ is denoted by $\lambda$. An equivalence relation $\mathscr{R}$ in $\lambda$ is defined by

$$
l_{1} \stackrel{\circ}{=} l_{2} \quad \text { if }\left.\left\{\left(l_{1}-l_{2}\right) g\right\}\right|_{g=0}=0 \quad \text { for any } g \in \gamma .
$$

The symbol $\left.\right|_{g=0}$ indicates the evaluation of a quantity under the condition $g=0$, $D_{x_{i}} g=0, D_{x_{i}} D_{x_{j}} g=0, \cdots$. It is not difficult to show:

Proposition 1. The operator (1) is equivalent to

$$
\begin{aligned}
l & =\hat{\zeta}^{\mu} \partial_{z^{\mu}}+\hat{\zeta}_{i}^{\mu} \partial_{z_{i}^{\mu}}+\hat{\zeta}_{i j}^{\mu} \partial_{z_{i j}^{\mu}}+\cdots, \\
\hat{\zeta}^{\mu} & =\zeta^{\mu}-z_{i}^{\mu} \xi^{i}, \quad \hat{\zeta}_{i \cdots j k}^{\mu}=D_{x_{k}} \hat{\zeta}_{i \cdots j}^{\mu}
\end{aligned}
$$

DEFINITION 1. The operator (1) is called a generator of an invariance group of an equation $f\left(\omega^{(n)}\right)=\left(f^{1}, f^{2}, \cdots, f^{K}\right)=0, f: w^{(n)} \rightarrow R^{K}, f^{i} \in \gamma$, when it satisfies the equality $\left.l \cdot f\right|_{f=0}=0$. In this case we say $f=0$ admits the generator $l$.

Any operator equivalent to a generator is also a generator for the same equation.

Since an operator of the form (4) is generally easier to work with than one of the form (1), we only consider operators of the form

$$
l=\theta^{\mu} \partial_{z^{\mu}}+\theta_{i}^{\mu} \partial_{z_{i}^{\mu}}+\theta_{i j}^{\mu} \partial_{z_{i j}^{\mu}}+\cdots,
$$

$\theta^{\mu} \in \gamma, \theta_{i \cdots j k}^{\mu}=D_{x_{k}} \theta_{i \cdots j \cdot}^{\mu}$. We write this operator simply as $l=\theta^{\mu} \partial_{z^{\mu}}$. An element of the quotient space $\lambda / \mathscr{R}$ is represented by $l=\theta^{\mu} \partial_{z^{\mu}}$.

If one associates functions $u^{\mu}(x), u_{i}^{\mu}(x), \cdots$ with $z^{\mu}, z_{i}^{\mu}, \cdots$, the equation $f\left(\omega^{(n)}\right)=0$ becomes a system of $K n$th order differential equations. Here it should be noted that Lie [4]-[6] established a systematic algorithm to find generators admitted by differential equations.

When the equation $f\left(\omega^{(n)}\right)=0$ is linear in $z, z, z, \cdots$, the equation is known to admit a generator associated with the superposition principle. To be consistent with 
the later discussion, we use capital letters $X, Z$ in the following lemma and proposition.

Proposition 2. A system of linear equations $\mathscr{A}_{\mu}^{\nu} Z^{\mu}-\Phi^{\nu}(X)=0, \nu=1,2, \cdots, K$, $K \leqq N, \Phi^{\nu}(X): R^{M} \rightarrow R$, with linear operator $\mathscr{A}_{\mu}^{\nu}$ defined by

$$
\mathscr{A}_{\mu}^{\nu} Z^{\mu}=A_{\mu}^{\nu}(X) Z^{\mu}+A_{\mu}^{\nu i}(X) Z_{i}^{\mu}+\cdots+A_{\mu}^{\nu i_{1} i_{2} \cdots i_{n}}(X) Z_{i_{1} i_{2} \cdots i_{n}}^{\mu},
$$

$A_{\mu}^{\nu i_{1} i_{2} \cdots i_{k}}(X): R^{M} \rightarrow R$, admits a generator $L=U^{\mu}(X) \partial_{Z^{\mu}}$ depending upon an arbitrary solution $\left\{U^{\mu}(X) ; \mu=1,2, \cdots, N\right\}$ of the system of linear differential equations $\mathscr{A}_{\mu}^{\nu} U^{\mu}(X)=0$, i.e.,

$$
A_{\mu}^{\nu}(X) U^{\mu}+A_{\mu}^{\nu i}(X) U_{i}^{\mu}+\cdots+A_{\mu}^{\nu i_{1} i_{2} \cdots i_{n}}(X) U_{i_{1} i_{2} \cdots i_{n}}^{\mu}=0,
$$

$\nu=1,2, \cdots, K$.

Here and in the following, $U_{i j \cdots k}^{\mu}=\partial_{X_{i}} \partial_{X_{j}} \cdots \partial_{X_{k}} U^{\mu}(X)$. It is easy to see that if $\mathscr{A}_{\mu}^{\nu} Z^{\mu}-\Phi^{\nu}(X)=0$, then $\bar{Z}=(1+c L) Z, c$ constant, corresponding to a superposition of $c U$ with $Z$, satisfies the equation $\mathscr{A}_{\mu}^{\nu} \bar{Z}^{\mu}-\Phi^{\nu}(X)=0$.

DEFINITION 2. An operator $L=U^{\mu}(X) \partial_{Z^{\mu}}$ is said to be a superposition operator of the equation $\mathscr{A}_{\mu}^{\nu} Z^{\mu}-\Phi^{\nu}(X)=0, \nu=1,2, \cdots, K$, if $\mathscr{A}_{\mu}^{\nu} U^{\mu}=0$.

It may happen that nonlinear equations can be resolved algebraically into linear equations. For example, the equation $\left(Z_{1}\right)^{2}-(Z)^{2}=0$ is solvable as $Z_{1} \pm Z=0$. We now define precisely the meaning of linear systems.

Definition 3. $K C^{1}$ functions $W^{(n)} \rightarrow R$

$$
F^{\nu}\left(\Omega^{(n)}\right)=F^{\nu}(X, Z, \underset{1}{Z}, \cdots, Z), \quad \nu=1,2, \cdots, K
$$

are functionally independent in the domain $D(W)$ if and only if there exist $K$ components of $\left(\underset{1}{Z}, Z_{2}, \cdots, Z\right)$, denoted by $Y^{1}, Y^{2}, \cdots, Y^{K}$, for which the Jacobian of $\left(F^{1}, F^{2}, \cdots, F^{K}\right)$ is nonzero:

$$
\frac{D\left(F^{1}, F^{2}, \cdots, F^{K}\right)}{D\left(Y^{1}, Y^{2}, \cdots Y^{K}\right)} \neq 0 \quad \text { in } D(W) .
$$

We denote by $D(W ; F=0)$ the set of points $\Omega \in D(W)$ satisfying the equations $F^{\nu}\left(\Omega^{(n)}\right)=0, \nu=1,2, \cdots, K$. The implicit function theorem ensures that if $\left\{F^{\nu}\left(\Omega^{(n)}\right)\right\}$ is a set of functionally independent functions and $D(W ; F=0)$ is nonempty, then in every neighborhood of $\Omega \in D(W ; F=0)$ there exists a unique set of $K C^{1}$ functions $\Psi^{\nu}\left(\Omega^{(n)}\right), \nu=1,2, \cdots, K$, independent of $Y^{1}, Y^{2}, \cdots, Y^{K}$, with the property that the functions $F^{\nu}\left(\Omega^{(n)}\right)$ all vanish with the substitutions $Y^{\nu}=\Psi^{\nu}\left(\Omega^{(n)}\right), \nu=1,2, \cdots, K$. We call $Y^{\nu}=\Psi^{\nu}\left(\Omega^{(n)}\right)$ an explicit form of the equations $F^{\nu}\left(\Omega^{(n)}\right)=0$.

DEFINITION 4. $K$ equations

$$
F^{\nu}\left(\Omega^{(n)}\right)=0, \quad \nu=1,2, \cdots, K, \quad K \leqq N,
$$

are said to be independent if and only if $\left\{F^{\nu}\right\}$ are functionally independent in a given domain $D(W)$ and $D(W ; F=0)$ is nonempty. A system of $K$ independent equations is called a linear system if and only if its explicit form is linear in $Z, Z_{1}, \cdots, Z_{n}$, namely,

$$
Y^{\nu}=\hat{\mathscr{A}}_{\mu}^{\nu} Z^{\mu}+\hat{\Phi}^{\nu}(X), \quad \nu=1,2, \cdots, K
$$

with

$$
\begin{gathered}
\hat{\mathscr{A}}_{\mu}^{\nu} Z^{\mu}=\hat{A}_{\mu}^{\nu}(X) Z^{\mu}+\hat{A}_{\mu}^{\nu i}(X) Z_{i}^{\mu}+\cdots+\hat{A}_{\mu}^{\nu i_{1} i_{2} \cdots i_{n}}(X) Z_{i_{1} i_{2} \cdots i_{n}}^{\mu}, \\
\hat{A}_{\mu}^{\nu}: R^{M} \rightarrow R, \quad \hat{A}_{\mu}^{\nu i_{1} i_{2} \cdots i_{k}}: R^{M} \rightarrow R, \quad \hat{\Phi}^{\nu}: R^{M} \rightarrow R .
\end{gathered}
$$


The following is obvious:

LEMMA 1. A linear system $F^{\nu}\left(\Omega^{(n)}\right)=0, \nu=1,2, \cdots, K$, admits a superposition operator $L=U^{\mu}(X) \partial_{Z^{\mu}}$ associated with its explicit form

$$
Y^{\nu}=\hat{\mathscr{A}}_{\mu}^{\nu} Z^{\mu}+\hat{\Phi}^{\nu}(X) \text {. }
$$

1.2. Contact transformations. We now consider a 1-1 transformation $T$ of the space $w$ to a space $W$ with coordinates $\Omega=(X, Z, Z, \cdots, Z, \cdots)$. We let $\Omega^{(n)}=$ $(X, Z, Z, \cdots, Z)$. As we associate with $z^{\nu}, z_{i}^{\nu}, \cdots$ functions $u^{\nu}(x), u_{i}^{\nu}(x), \cdots$ and with $Z^{\nu}, Z_{i}^{\nu}, \cdots$ functions $U^{\nu}(X), U_{i}^{\nu}(X), \cdots$, it is necessary to impose a condition that the transformation $T$ preserve the contact conditions:

$$
\begin{aligned}
& d z^{\nu}-z_{i}^{\nu} d x_{i}=0, \\
& d z_{i_{1} \cdots i_{k}}^{\nu}-z_{i_{1} \cdots i_{k} i_{k+1}}^{\nu} d x_{i_{k+1}}=0, \\
& \quad \downarrow^{T} \\
& d Z^{\nu}-Z_{i}^{\nu} d X_{i}=0, \\
& d Z_{i_{1} \cdots i_{k}}^{\nu}-Z_{i_{1} \cdots i_{k} i_{k+1}}^{\nu} d X_{i_{k+1}}=0,
\end{aligned}
$$

$\nu=1,2, \cdots, N, i_{j}=1,2, \cdots, M, k=1,2, \cdots$. We call a transformation $T$ satisfying these conditions a contact transformation.

Bäcklund [7] proved that for a scalar $z$ the most general 1-1 transformation $\omega \leftrightarrow \Omega$ with this property is the extended contact transformation of Lie. On the other hand, for a vector $z$, the most general 1-1 transformation satisfying (8) is the extendedpoint transformation [8]. We summarize the basic properties of a Lie contact transformation.

Contact transformation of Lie. The following definition [9] of the Lie contact transformation is most suitable for our purposes:

A transformation $T: w^{(1)} \rightarrow W^{(1)}$

$$
X=\bar{X}(x, z, z), \quad Z=\bar{Z}(x, z, z), \quad \underset{1}{Z}=\bar{Z}_{1}(x, z, z), \quad z, Z \in R,
$$

$\bar{X}: w^{(1)} \rightarrow R^{M}, \bar{Z}: w^{(1)} \rightarrow R, \bar{Z}: w^{(1)} \rightarrow R^{M}$, is a Lie contact transformation, i.e.,

$$
d Z-Z_{i} d X_{i}=\rho\left(\omega^{(1)}\right)\left(d z-z_{i} d x_{i}\right)
$$

if and only if

1) $\bar{X}_{1}, \bar{X}_{2}, \cdots, \bar{X}_{M}$ and $\bar{Z}$ are independent functions of $x, z, z$ and satisfy $\left[\bar{X}_{i}, \bar{X}_{j}\right]=0,\left[\bar{Z}, \bar{X}_{i}\right]=0, i, j=1,2, \cdots, M$.

2) $\bar{Z}_{1}, \bar{Z}_{2}, \cdots, \bar{Z}_{M}$ are determined from

$$
\partial_{x_{i}} \bar{Z}+z_{i} \partial_{z} \bar{Z}=Z_{j}\left(\partial_{x_{i}} \bar{X}_{j}+z_{i} \partial_{z} \bar{X}_{j}\right)
$$

or from

$$
\partial_{z_{i}} \bar{Z}=Z_{j} \partial_{z_{i}} \bar{X}_{j}
$$

and $\rho\left(\omega^{(1)}\right)$ from

$$
\rho=\partial_{z} \bar{Z}-\bar{Z}_{i} \partial_{z} \bar{X}_{i}=\left[\bar{X}_{k}, \bar{Z}_{k}\right], \quad k=1,2, \cdots, M .
$$

The Lagrange bracket $[\cdot, \cdot]$ of two functions $\phi\left(\omega^{(1)}\right)$ and $\psi\left(\omega^{(1)}\right)$ is defined by

$$
[\phi, \psi]=\left(\partial_{z_{i}} \phi\right)\left(\partial_{x_{i}} \psi+z_{i} \partial_{z} \psi\right)-\left(\partial_{z_{i}} \psi\right)\left(\partial_{x_{i}} \phi+z_{i} \partial_{z} \phi\right) .
$$


Extensions of (9) to higher coordinates $\underset{n}{Z}, n>1$, are found from the contact condition (8). We write the extension of (9) to $\Omega^{(m)}$ and its inverse as

$$
\Omega^{(m)}=\bar{\Omega}^{(m)}\left(\omega^{(m)}\right), \quad \omega^{(m)}=\bar{\omega}^{(m)}\left(\Omega^{(m)}\right)
$$

and the infinite extensions as

$$
\Omega=\bar{\Omega}(\omega), \quad \omega=\bar{\omega}(\Omega) .
$$

It is convenient to write the right-hand sides of (15) as

$$
\bar{\Omega}^{(m)}\left(\omega^{(m)}\right) \equiv T \omega^{(m)}, \quad \bar{\omega}^{(m)}\left(\Omega^{(m)}\right) \equiv T^{-1} \Omega^{(m)}
$$

and those of (16)

$$
\bar{\Omega}(\omega) \equiv T \omega, \quad \bar{\omega}(\Omega) \equiv T^{-1} \Omega .
$$

1.3. Transformations of $f$ and $l$ by 1-1 contact transformations. The cases of a scalar $z$ and a vector $z$ are discussed separately since they admit different types of $1-1$ contact transformations.

a) Scalar $z$. We assume that the transformation (16) is analytic in $D(w)$. We let $D(W)$ be the image of $D(w)$ by (16). $\Gamma$ denotes the space of functions $W^{(k)} \rightarrow R, k$ finite, analytic in $D(W)$ and $\Lambda$ is the space of operations $L=\Theta \partial_{z}, \Theta \in \Gamma$.

The transformation of a scalar function $f\left(\omega^{(n)}\right) \in \gamma \mathrm{y}$ the Lie contact transformation $T$ is written as $T f\left(\omega^{(n)}\right)$ and defined by

$$
T f\left(\omega^{(n)}\right) \equiv f\left(T^{-1} \Omega^{(n)}\right) \in \Gamma .
$$

The inverse transformation of $F\left(\Omega^{(n)}\right)$ into $\gamma$ is defined by

$$
T^{-1} F\left(\Omega^{(n)}\right)=F\left(T \omega^{(n)}\right) \in \gamma .
$$

Under the change of variables $\omega \rightarrow \Omega$ defined by (9) and its extension, the operator (5), i.e., $l=\theta \partial_{z}+\theta_{i} \partial_{z_{i}}+\cdots$ is transformed to

$$
L=\left(l \bar{X}_{i}\right) \partial_{X_{i}}+(l \bar{Z}) \partial_{Z}+\left(l \bar{Z}_{i}\right) \partial_{Z_{i}}+\cdots .
$$

We write $L=T l T^{-1} \in \Lambda$. The equivalence relation in $\Lambda$ is defined as in $\lambda$. It is clear that if $l_{1} \stackrel{\varrho}{=} l_{2}$, then $L_{1} \stackrel{\varrho}{=} L_{2}$. Using Proposition 1 and the equalities (12) and (13), we find that

$$
\begin{aligned}
T \theta\left(\omega^{(m)}\right) \partial_{z} T^{-1} \stackrel{\varrho}{=}\left[\rho\left(T^{-1} \Omega^{(1)}\right) \theta\left(T^{-1} \Omega^{(m)}\right)\right] \partial_{Z}, \\
T^{-1} \Theta\left(\Omega^{(m)}\right) \partial_{Z} T \stackrel{\varrho}{=}\left[\left\{\rho\left(\omega^{(1)}\right)\right\}^{-1} \Theta\left(T \omega^{(m)}\right)\right] \partial_{z} .
\end{aligned}
$$

If $f=0$ admits a generator $l$, then any operator equivalent to $T l T^{-1}$ is a generator of an invariance group of $T f=0$. Conversely, if $L$ is a generator of $F=0$, an operator $l \stackrel{\circ}{\triangleq} T^{-1} L T$ is a generator of $T^{-1} F=0$. Thus, in view of (22), we have:

LEMMA 2. An operator $L=\Theta\left(\Omega^{(m)}\right) \partial_{z}$ is an invariance group generator of the equation $F\left(\Omega^{(n)}\right)=0$ if and only if the generator $l=\left[\left\{\rho\left(\omega^{(1)}\right)\right\}^{-1} \Theta\left(T \omega^{(m)}\right)\right] \partial_{Z}$ is admitted by the equation $T^{-1} F \equiv f\left(\omega^{(n)}\right)=0$.

b) Vector $z$. In this case the most general 1-1 contact transformation $w \rightarrow W$ is the extended point transformation ${ }^{1}$ of

$$
X=\bar{X}(x, z), \quad Z=\bar{Z}(x, z),
$$

\footnotetext{
${ }^{1}$ In the present context the canonical transformations of classical mechanics $T=t, P=\bar{P}(p, q, t)$, $Q=\bar{Q}(p, q, t)$ correspond to point transformations where $x=t,\left(z^{1}, z^{2}, \cdots, z^{n}\right)=p,\left(z^{n+1}, z^{n+2}, \cdots, z^{2 n}\right)=$ $q$.
} 
$\bar{X}: R^{M+N} \rightarrow R^{M}, \bar{Z}: R^{M+N} \rightarrow R^{N}$. Transformations of $Z$ are determined from (23) by (8). As in Lie contact transformations, we write the extension of $(23)$ as $\Omega^{(m)}=$ $\bar{\Omega}^{(m)}\left(\omega^{(m)}\right) \equiv T \omega^{(m)}$ and the inverse as $\omega^{(m)}=\bar{\omega}^{(m)}\left(\Omega^{(m)}\right) \equiv T^{-1} \Omega^{(m)}$. A transformation of a vector function $f\left(\omega^{(n)}\right)$ is defined by (19) and that of $F\left(\Omega^{(n)}\right)$ by $\left(19^{\prime}\right)$. Transformations of operators are defined in the same way as above. In particular,

$$
T^{-1} \Theta^{\mu}\left(\Omega^{(m)}\right) \partial_{Z^{\mu}} T \stackrel{\circ}{=}\left[\Theta^{\mu}\left(T \omega^{(m)}\right) \sigma_{\mu}^{\nu}\left(\omega^{(1)}\right)\right] \partial_{z^{\nu}}
$$

with

$$
\sigma_{\mu}^{\nu}\left(\omega^{(1)}\right)=\partial_{Z^{\mu}} \bar{z}^{\nu}-z_{i}^{\nu} \partial_{Z^{\mu}} \bar{x}_{i},
$$

where $x=\bar{x}(X, Z), z=\bar{z}(X, Z)$ define the inverse of (23), and corresponding to Lemma 2, we have

LEMMA 3. An operator $L=\Theta^{\mu}\left(\Omega^{(m)}\right) \partial_{Z^{\mu}}$ is an invariance group generator of the equation $F\left(\Omega^{(n)}\right)=0$ if and only if $T^{-1} F \equiv f\left(\omega^{(n)}\right)=0$ admits (24).

2. 1-1 mappings between nonlinear and linear equations. We now show that the group analysis of a given nonlinear differential equation enables us to determine whether the equation is transformable to a linear equation by a 1-1 mapping. First, for a scalar equation we have:

THEOREM 1. A scalar $n$th order nonlinear equation

$$
f\left(\omega^{(n)}\right)=f(x, z, \underset{1}{z}, \underset{2}{z}, \cdots, \underset{n}{z})=0, \quad x \in R^{M}, \quad z \in R,
$$

is transformable by a 1-1 contact transformation to a linear equation if and only if the equation $f=0$ admits a generator $l$ of the form

$$
l=\left[\sigma\left(\omega^{(1)}\right) U\left(\bar{X}\left(\omega^{(1)}\right)\right)\right] \partial_{z}
$$

where equation

1) $U(X): R^{M} \rightarrow R$ is an arbitrary solution of some $n$th order linear differential

$$
\mathscr{A} U=A(X) U+A^{i}(X) U_{i}+\cdots+A^{i_{1} i_{2} \cdots i_{n}}(X) U_{i_{1} i_{2} \cdots i_{n}}=0,
$$

2) $\bar{X}\left(\omega^{(1)}\right): R^{2 M+1} \rightarrow R^{M}$ is a component of a Lie contact transformation

$$
X=\bar{X}\left(\omega^{(1)}\right), \quad Z=\bar{Z}\left(\omega^{(1)}\right), \quad \underset{1}{Z}=\bar{Z}\left(\omega^{(1)}\right)
$$

and

$$
\sigma\left(\omega^{(1)}\right)=\left[\rho\left(\omega^{(1)}\right)\right]^{-1}=\left(\partial_{z} \bar{Z}-\bar{Z}_{i} \partial_{z} \bar{X}_{i}\right)^{-1} .
$$

The transformation (29) maps equation (26) to a linear equation $\mathscr{A} Z-\Phi(X)=0, \mathscr{A}$ defined by (28).

Proof. Suppose that equation (26) is transformable to a linear equation by an extended Lie contact transformation of (9). By Lemma 1, this linear equation admits the superposition generator $L=U(X) \partial_{Z}$ of the equation $\mathscr{A} Z-\Phi(X)=0$. Hence, according to Lemma 2, equation (26) must admit (27).

Conversely, suppose that (26) admits a generator of the form (27) with properties 1) and 2). The transformed equation of (26) by (29) is written as $T f \equiv F\left(X, Z, Z_{1}, \cdots, Z_{n}\right)=0$. In view of Lemma $2, F=0$ admits the generator $L=$ $U(X) \partial_{Z}$. Thus,

$$
L F=F_{Z} U+F_{Z_{i}} U_{i}+\cdots+F_{Z_{i_{1} i_{2} \cdots i_{n}}} U_{i_{1} i_{2} \cdots i_{n}}=0
$$


for any $\Omega^{(n)}$ satisfying $F\left(\Omega^{(n)}\right)=0$ and for any $U(X)$ satisfying the differential equation (28). It is easy to show that (28) and (31) involve the same set of $U, U_{i}, \cdots$. We assume without a loss of generality that both contain $U$. Eliminating $U$ between the two equations, we get

$$
0=\left(A F_{Z_{i}}-A^{i} F_{Z}\right) U_{i}+\left(A F_{Z_{i j}}-A^{i j} F_{Z}\right) U_{i j}+\cdots .
$$

Since $U$ represents an arbitrary solution of (28), at any point $X$ an arbitrary set of values may be assigned to $U_{i}, U_{i j}, \cdots$, and thus all the coefficients in (32) must vanish. This is possible only if $F$ has the form $G(\mathscr{A Z}, X), G$ arbitrary. Therefore, (29) maps (26) to an equation $G(\mathscr{A} Z, X)=0$, which is solvable in the explicit form $\mathscr{A} Z-\Phi(X)=$ 0 .

In this theorem, $z, z_{i}, \cdots, Z, Z_{i}, \cdots$ are considered to be coordinates of the spaces $w, W$. Because of the contact condition (8) imposed upon $T$ this theorem implies:

THEOREM 2. A scalar nonlinear differential equation

$$
f\left(x, u, \underset{1}{u}, \underset{2}{u}, \cdots, u_{n}\right)=0, \quad x \in R^{M}, \quad u: R^{M} \rightarrow R
$$

is transformable to a linear differential equation by a 1-1 mapping if and only if the equation $f(x, z, \underset{1}{z}, \underset{2}{z}, \cdots, \underset{n}{z})=0$ admits a generator of the form (27). The mapping is given by (29) and it transforms equation (33) to a differential equation which is solvable in an explicit form

$$
\mathscr{A} U-\Phi(X)=0
$$

We now turn to a vector equation.

THEOREM 3. A system of $K$ independent $n$th order nonlinear equations

$$
f^{\nu}\left(\omega^{(n)}\right)=f^{\nu}(x, z, \underset{1}{z}, \underset{2}{z}, \cdots, \underset{n}{z})=0, \quad \nu=1,2, \cdots, K, \quad K \leqq N,
$$

$x \in R^{M}, z \in R^{N}$, is transformable by a 1-1 contact transformation to a linear system if and only if the system $\left\{f^{\nu}=0\right\}$ admits a generator of the form

$$
l=\left[U^{\mu}(\bar{X}(x, z)) \sigma_{\mu}^{\nu}(x, z, z)\right] \partial_{z^{\nu}}
$$

where

1) $U^{\mu}(X), \mu=1,2, \cdots, N$, is an arbitrary solution of some system of $n$th order linear differential equations

$$
\mathscr{A}_{\mu}^{\nu} U^{\mu}=A_{\mu}^{\nu}(X) U^{\mu}+A_{\mu}^{\nu i}(X) U_{i}^{\mu}+\cdots+A_{\mu}^{\nu i_{1} i_{2} \cdots i_{n}}(X) U_{i_{1} i_{2} \cdots i_{n}}^{\mu}=0,
$$

$\nu=1,2, \cdots, K$, and

2) $\bar{X}(x, z): R^{M+N} \rightarrow R^{M}$ is a component of a point transformation

$$
X=\bar{X}(x, z), \quad Z=\bar{Z}(x, z)
$$

with inverse transformation $x=\bar{x}(X, Z), z=\bar{z}(X, Z)$ and

$$
\sigma_{\mu}^{\nu}=\left[\partial_{Z^{\mu}} \bar{z}^{\nu}-z_{i}^{\nu} \partial_{Z^{\mu}} \bar{x}_{i}\right]_{Z=\bar{X}}
$$

The extended point transformation of (38) maps (35) to a linear system with explicit forms

$$
\mathscr{A}_{\mu}^{\nu} Z^{\mu}-\Phi^{\nu}(X)=0, \quad \nu=1,2, \cdots, K \text {. }
$$


Proof. Recalling that the most general 1-1 contact transformation in the case of a vector $z$ is the extended point transformation, we suppose that there exists an extended point transformation

$$
X=\bar{X}(x, z), \quad Z=\bar{Z}(x, z), \quad \underset{1}{Z}=\bar{Z}(x, z, z), \quad \cdots,
$$

mapping (35) to a linear system (40). By Lemma 1, this linear system admits the superposition generator $L=U^{\mu}(X) \partial_{Z^{\mu}}$. In view of Lemma 3, the system (35) must admit the generator (36) with properties 1 ) and 2).

Conversely, suppose (35) admits the generator (36). Under transformation (38), the generator (36) is transformed into

$$
L=U^{\mu}(X) \partial_{Z^{\mu}}
$$

and (35) into, say, $F^{\nu}(X, Z, Z, \cdots, Z)=0, \nu=1,2, \cdots, K$. The system $\left\{F^{\nu}=0\right\}$ is solvable in explicit forms for $K$ of the $Z_{1}, Z_{2}, \cdots, Z_{n}$. Without loss of generality, for these $K$ components we choose $Z_{1}^{\nu}, \nu=1,2, \cdots, K$ and write the explicit forms as

$$
Z_{1}^{\nu}+\phi^{\nu}(X, Z, Z, \cdots, Z)=0, \quad \nu=1,2, \cdots, K,
$$

where $\phi^{\nu}$ are independent of $Z_{1}^{\mu}, \mu=1,2, \cdots, K$. According to Lemma 3, the system $\left\{F^{\nu}=0\right\}$ admits the generator (42), and hence so does the system (43). Thus

$$
U_{1}^{\nu}+\phi_{Z^{\mu}}^{\nu} U^{\mu}+\phi_{Z_{i}^{\mu}}^{\nu} U_{i}^{\mu}+\cdots+\phi_{Z_{i_{1} i_{2} \cdots i_{n}}^{\mu}}^{\nu} U_{i_{1} i_{2} \cdots i_{n}}^{\mu}=0, \quad \nu=1,2, \cdots, K,
$$

where $\phi_{Z^{\mu}}^{\nu}=\partial_{Z^{\mu}} \phi^{\nu}$, etc. This holds for any $U(X)$ satisfying the differential equation

$$
A_{\mu}^{\nu}(X) U^{\mu}+A_{\mu}^{\nu i}(X) U_{i}^{\mu}+\cdots+A_{\mu}^{\nu i_{1} i_{2} \cdots i_{n}}(X) U_{i_{1} i_{2} \cdots i_{n}}^{\mu}=0, \quad \nu=1,2, \cdots, K .
$$

It is easy to see that (44) can involve only those $U^{\mu}, U_{i}^{\mu}, \cdots$ appearing in (45). Equation (45) is solvable for $U_{1}^{\mu}, \mu=1,2, \cdots, K$. This is seen as follows. Suppose this is not the case, i.e., rank $\left|A_{\mu}^{\nu 1}\right|<K, \mu, \nu \leqq K$. We fix a point $X=X_{0}$ and assign to $U^{\nu}\left(X_{0}\right), U_{i}^{\mu}\left(X_{0}\right)$ [either $i=1, \mu>K$ or $i>1$ ] $, U_{i j}^{\nu}\left(X_{0}\right), \cdots$ a set of values consistent with (45). For this set of values, there exist nonunique values of $U_{1}^{\nu}\left(X_{0}\right), \nu \leqq K$, satisfying (45) because of the above rank condition. On the other hand, the introduction of the same set of values into (44) uniquely determines the values of $U_{1}^{\nu}\left(X_{0}\right)$. This contradicts the condition that (44) holds for any solution of (45). Thus, (45) is solvable as

$$
U_{1}^{\nu}+\hat{A}_{\mu}^{\nu} U^{\mu}+\hat{A}_{\mu}^{\nu i} U_{i}^{\mu}+\cdots+\hat{A}_{\mu}^{\nu i_{1} i_{2} \cdots i_{n}} U_{i_{1} i_{2} \cdots i_{n}}^{\mu}=0, \quad \nu=1,2, \cdots, K
$$

Eliminating $U_{1}^{\nu}$ from (44) and (46), we have

$$
0 \equiv\left(\phi_{Z^{\mu}}^{\nu}-\hat{A}_{\mu}^{\nu}\right) U^{\mu}+\left(\phi_{Z_{i}^{\mu}}^{\nu}-\hat{A}_{\mu}^{\nu i}\right) U_{i}^{\mu}+\cdots+\left(\phi_{Z_{i_{1} i_{2} \cdots i_{n}}^{\nu}}^{\nu}-\hat{A}_{\mu}^{\nu i_{1} i_{2} \cdots i_{n}}\right) U_{i_{1} i_{2} \cdots i_{n}}^{\mu} .
$$

This is possible only if

$$
\phi^{\nu}=\hat{A}_{\mu}^{\nu} Z^{\mu}+\hat{A}_{\mu}^{\nu i} Z_{i}^{\mu}+\cdots+\hat{A}_{\mu}^{\nu i_{1} i_{2} \cdots i_{n}} Z_{i_{1} i_{2} \cdots i_{n}}^{\mu}+\hat{\Phi}^{\nu}(X)
$$

with $\hat{A}_{\mu}^{\nu 1}=0, \mu \leqq K$, and consequently the explicit form (43) of the transformed system $\left\{F^{\nu}=0\right\}$ is linear. It is also clear that (43) is equivalent to (40).

Recalling again that the most general 1-1 contact transformation in this case is the extended point transformation, we can deduce from this theorem that:

THEOREM 4. A system of $K$ independent nonlinear differential equations

$$
f^{\nu}(x, u, \underset{1}{u}, \cdots, \underset{n}{u})=0, \quad \nu=1,2, \cdots, K, \quad K \leqq N,
$$


$x \in R^{M}, u: R^{M} \rightarrow R^{N}$, is transformable by a 1-1 mapping to a system of linear differential equations if and only if the system $f^{\nu}(x, z, \underset{1}{z}, \cdots, \underset{n}{z})=0$ admits a generator of the form (36). The mapping is given by (38) and it transforms (47) to a system solvable in explicit form as

$$
\mathscr{A}_{\mu}^{\nu} U^{\mu}-\Phi^{\nu}(X)=0, \quad \nu=1,2, \cdots, K .
$$

These theorems ensure that if a given nonlinear system is transformable to a linear system by a 1-1 mapping, one can always find the mapping by examining the nature of the invariance group of the nonlinear system. The type of group to be considered depends on the number of dependent variables.

For a scalar equation we need only consider generators $l$ of the form

$$
l=\theta(x, z, \underset{1}{z}) \partial_{z}
$$

since any scalar nonlinear differential equation transformable to a linear equation admits an $l$ of the form (27). It should be emphasized that the function $\bar{X}\left(\omega^{(1)}\right)$, the factor $\sigma\left(\omega^{(1)}\right)$ and the linear differential equation (28) can all be found by examining the generators of the invariance group of (26). Once $\bar{X}$ is obtained, the function $\bar{Z}\left(\omega^{(1)}\right)$ is determined from the condition $\left[\bar{Z}, \bar{X}_{i}\right]=0$ which represents a system of first order partial differential equations for $\bar{Z}$. At this point, $\bar{Z}$ still admits functional arbitrariness. From $\bar{X}$ and $\bar{Z}$ we determine $\bar{Z}$ using conditions (11) or (12). Next we use (13) for the known $\rho=\sigma^{-1}$ to limit the arbitrariness in $\bar{Z}$. The resulting transformation $X=\bar{X}, Z=\bar{Z}, \underset{1}{Z}=\bar{Z}$ maps the nonlinear equation to an equation reducible to a linear equation $\mathscr{A} U-\Phi(X)=0$. The form of $\Phi(X)$ depends upon the remaining arbitrariness in $\bar{Z}$.

For a system of equations, because of the forms of (36) and (39), the generators $l$ to be considered are linear in $\left\{z_{i}^{\nu}\right\}$ :

$$
l=\left(\zeta^{\nu}(x, z)-z_{i}^{\nu} \xi^{i}(x, z)\right) \partial_{z^{\nu}} .
$$

In view of Proposition 1, we see that these are equivalent to generators of a point group

$$
l=\xi^{i}(x, z) \partial_{x_{i}}+\zeta^{\nu}(x, z) \partial_{z^{\nu}} .
$$

If there exists a mapping to a linear system, we can find the functions $\bar{X}(x, z)$, $\sigma_{\nu}^{\mu}(x, z, z)$ by comparing the resulting generators $\left(49^{\prime}\right)$ with (36). The functions $\bar{Z}^{\mu}(x, z)$ are to be determined from these functions using equations (39). Equation (37) is found on determining the invariance group.

Remark 1. It is possible for differential equations to admit generators whose forms are more general than those of (27) or (36) with the forms (27) or (36) as special cases. The Monge-Ampère equations considered in the next section are such examples. A system of ordinary differential equations also admits such generators.

3. Examples. To illustrate the use of our theorems, we consider some well-known equations transformable to linear equations. Since the linearization of differential equations $f^{\nu}(x, u, \underset{1}{u}, \cdots, \underset{n}{u})=0$ is equivalent to that of the equations $f^{\nu}(x, z, \underset{1}{z}, \cdots, \underset{n}{z})=$ 0 by a contact transformation, we only deal with the latter. In the following examples, where convenient, we let $x_{1}=x, x_{2}=y$ and adopt the customary notations $z_{x}=p$, $z_{y}=q, z_{x x}=r, z_{x y}=s, z_{y y}=t$. 
3.1. The equation $z_{x x}+\frac{1}{2}\left(z_{x}\right)^{2}-z_{y}=0$. We consider the equation

$$
f=z_{x x}+\frac{1}{2}\left(z_{x}\right)^{2}-z_{y}=0 \text {. }
$$

Assuming the form $l=\theta\left(x, y, z, z_{x}, z_{y}\right) \partial_{z}$ for a generator and applying Lie's algorithm, we find that $(50)$ admits

$$
\begin{gathered}
l_{1}=\left(y^{2} z_{y}+y x z_{x}+\frac{1}{2} x^{2}+y\right) \partial_{z}, \quad l_{2}=\left(y z_{y}+\frac{1}{2} x z_{x}\right) \partial_{z}, \\
l_{3}=z_{y} \partial_{z}, \quad l_{4}=z_{x} \partial_{z}, \quad l_{5}=\partial_{z}, \\
l_{6}=\left(y z_{x}+x\right) \partial_{z}, \quad l_{7}=U(x, y) e^{-z / 2} \partial_{z},
\end{gathered}
$$

where, in the generator $l_{7}, U(x, y)$ is an arbitrary solution of the heat equation $\mathscr{A} U \equiv U_{x x}-U_{y}=0$. This indicates that equation (50) is equivalent to a linear equation. To find the mapping, we compare $l_{7}$ with (27) to get $\bar{X}=x, \bar{Y}=y$ and $\sigma=e^{-z / 2}$. From the conditions $[\bar{X}, \bar{Z}]=[\bar{Y}, \bar{Z}]=0$, we obtain $\bar{Z}=\bar{Z}(x, y, z)$. Thus, the mapping is a point transformation. From (13) and $\rho=(\sigma)^{-1}=e^{z / 2}$, we find that $\partial_{z} \bar{Z}=e^{z / 2}$. The mapping is then

$$
X=x, \quad Y=y, \quad Z=2 e^{z / 2}+h(x, y)
$$

where $h(x, y)$ is an arbitrary function of $x$ and $y$. It is easy to check that the extended point transformation of (52) maps equation (50) to the linear equation

$$
\mathscr{A} Z-\Phi(X, Y) \equiv Z_{X X}-Z_{Y}-\left(h_{X X}-h_{Y}\right)=0 \text {. }
$$

Setting $h=0$, from Theorem 2 we see that the transformation

$$
X=x, \quad Y=y, \quad U=2 e^{u / 2}
$$

maps the differential equation $u_{x x}+\frac{1}{2}\left(u_{x}\right)^{2}-u_{y}=0$ to the equation $\mathscr{A} U=U_{X X}-U_{Y}=0$, and moreover the inverse of $(54)$,

$$
x=X, \quad y=Y, \quad u=2 \ln \left(\frac{1}{2} U\right)
$$

defines an implicit solution $u(x, y)$ of this nonlinear differential equation for any solution $U(X, Y)$ of $U_{X X}-U_{Y}=0$. In this case the explicit form is

$$
u=2 \ln \left(\frac{1}{2} U(x, y)\right) \text {. }
$$

3.2. Hodograph transformations. In this example we let $z^{1}=w, z^{2}=v$ and consider a system of quasilinear equations

$$
f^{i}=a^{i x}(w, v) w_{x}+a^{i y}(w, v) w_{y}+b^{i x}(w, v) v_{x}+b^{i y}(w, v) v_{y}=0, \quad i=1,2,
$$

where $a^{i x}, a^{i y}, b^{i x}$ and $b^{i y}$ are functions of $w, v$. For the invariance group of this equation we have:

Proposition 4. Provided $J=w_{x} v_{y}-v_{x} w_{y} \neq 0$, the system (57) admits a generator of the form

$$
l=-\left\{U^{1}(w, v) w_{x}+U^{2}(w, v) w_{y}\right\} \partial_{w}-\left\{U^{1}(w, v) v_{x}+U^{2}(w, v) v_{y}\right\} \partial_{v},
$$

where $\left\{U^{1}(w, v), U^{2}(w, v)\right\}$ is an arbitrary solution of the system of linear differential equations

$$
\mathscr{A}_{\mu}^{i} U^{\mu}=b^{i y}(w, v) U_{w}^{1}-a^{i y}(w, v) U_{v}^{1}-b^{i x}(w, v) U_{w}^{2}+a^{i x}(w, v) U_{v}^{2}=0, \quad i=1,2,
$$

with $U_{w}^{i}=\partial_{w} U^{i}, U_{v}^{i}=\partial_{v} U^{i}$. 
Proof. Since $D_{x} f^{i}=D_{y} f^{i}=0$, we find that

$$
\begin{aligned}
-l \cdot f^{i}= & w_{x} U_{w}^{1}\left(a^{i x} w_{x}+a^{i y} w_{y}\right)+v_{y} U_{v}^{2}\left(b^{i x} v_{x}+b^{i y} v_{y}\right) \\
& +w_{y} U_{w}^{2}\left(a^{i x} w_{x}+a^{i y} w_{y}\right)+v_{x} U_{v}^{1}\left(b^{i x} v_{x}+b^{i y} v_{y}\right) \\
& +v_{x} U_{w}^{1}\left(b^{i x} w_{x}+b^{i y} w_{y}\right)+w_{y} U_{v}^{2}\left(a^{i x} v_{x}+a^{i y} v_{y}\right) \\
& +v_{y} U_{w}^{2}\left(b^{i x} w_{x}+b^{i y} w_{y}\right)+w_{x} U_{v}^{1}\left(a^{i x} v_{x}+a^{i y} v_{y}\right) .
\end{aligned}
$$

Using equation (57) in the first two rows of this expression, we find that

$$
\left.l \cdot f^{i}\right|_{\substack{f_{1}=0 \\ f_{2}=0}}=J \cdot\left(b^{i y} U_{w}^{1}-a^{i y} U_{v}^{1}-b^{i x} U_{w}^{2}+a^{i x} U_{v}^{2}\right)
$$

which vanishes from (59).

To construct the mapping to a linear equation, we compare (58) with (36) which in the present case has the form

$$
l=\left\{U^{1}(\bar{X}, \bar{Y}) \sigma_{1}^{1}+U^{2}(\bar{X}, \bar{Y}) \sigma_{2}^{1}\right\} \partial_{w}+\left\{U^{1}(\bar{X}, \bar{Y}) \sigma_{1}^{2}+U^{2}(\bar{X}, \bar{Y}) \sigma_{2}^{2}\right\} \partial_{v} .
$$

Clearly $\bar{X}=w, \bar{Y}=v, \sigma_{1}^{1}=-w_{x}, \sigma_{2}^{1}=-w_{y}, \sigma_{1}^{2}=-v_{x}, \sigma_{2}^{2}=-v_{y}$. The definition of $\sigma_{\mu}^{\nu}$ leads to $\partial_{W} \bar{x}=1, \partial_{V} \bar{x}=0, \partial_{W} \bar{y}=0, \partial_{V} \bar{y}=1$. Thus we have a solution $\bar{x}=W$, $\bar{y}=V$. Combining these, we find the hodograph transformation

$$
x=W, \quad y=V, \quad w=X, \quad v=Y,
$$

which is well known [10] to map the equation (57) to a linear system

$$
\mathscr{A}^{i} Z=b^{i y}(X, Y) W_{X}-a^{i y}(X, Y) W_{Y}-b^{i x}(X, Y) V_{X}+a^{i x}(X, Y) V_{Y}=0
$$
with $Z=(W, V)$.

3.3. The Legendre transformation. We consider a second order quasilinear equation

$$
f=a(p, q) r+2 b(p, q) s+c(p, q) t=0,
$$

where $a, b$ and $c$ are functions of $p$ and $q$. We have:

PROPOSITION 5. Equation (62) admits a generator $l=U(p, q) \partial_{z}$ with an arbitrary solution $U(p, q)$ of the linear differential equation

$$
\mathscr{A} U=a(p, q) U_{q q}-2 b(p, q) U_{p q}+c(p, q) U_{p p}=0,
$$

where $U_{q q}=\left(\partial_{q}\right)^{2} U, U_{p q}=\partial_{p} \partial_{q} U, U_{p p}=\left(\partial_{p}\right)^{2} U$.

Proof. On introducing $w=p, v=q, w_{x}=r, v_{y}=t, w_{y}=v_{x}=s,(62)$ is equivalent to the system

$$
\begin{aligned}
& a(w, v) w_{x}+b(w, v)\left(w_{y}+v_{x}\right)+c(w, v) v_{y}=0, \\
& w_{y}-v_{x}=0 .
\end{aligned}
$$

By Proposition 4, this system admits the generator given by (58) with arbitrary solution $\left\{U^{1}, U^{2}\right\}$ of the linear differential equations

$$
\begin{aligned}
& c(w, v) U_{w}^{1}-b(w, v)\left(U_{v}^{1}+U_{w}^{2}\right)+a(w, v) U_{v}^{2}=0, \\
& U_{v}^{1}-U_{w}^{2}=0 .
\end{aligned}
$$

Equation (67) allows us to introduce a function $U(w, v)$ for which

$$
U^{1}=\partial_{w} U \equiv U_{w}, \quad U^{2}=\partial_{v} U \equiv U_{v},
$$


and then (66) takes the form

$$
a U_{v v}-2 b U_{w v}+c U_{w w}=0 .
$$

Introducing (68) into (58) and using (65), we find that

$$
l \stackrel{\circ}{\triangleq}\left(D_{x} U\right) \partial_{w}+\left(D_{y} U\right) \partial_{v}
$$

Recalling that $w=p=z_{x}$ and $v=q=z_{y}$, we see that this is the first extended part of the operator $l=U(p, q) \partial_{z}$, and hence follows the assertion.

To construct a mapping of (62) to a linear equation we compare $l=-U(p, q) \partial_{z}$ with (27). Clearly, $\bar{X}=p, \bar{Y}=q$ and $\sigma=1$. To find $\bar{Z}$, we use $[\bar{Z}, \bar{X}]=[\bar{Z}, \bar{Y}]=0$, i.e.,

$$
\bar{Z}_{x}+p \bar{Z}_{z}=0, \quad \bar{Z}_{y}+q \bar{Z}_{z}=0
$$

The solution is $\bar{Z}=\bar{Z}(p, q, \alpha), \alpha=-z+p x+q y$. From (12), we get

$$
\bar{Z}_{p}+x \bar{Z}_{\alpha}=P, \quad \bar{Z}_{q}+y \bar{Z}_{\alpha}=Q
$$

with $P=Z_{X}$ and $Q=Z_{Y}$. Now from (30) with $\sigma=1$, we get $\bar{Z}_{\alpha}=1$, and consequently $\bar{Z}=-z+p x+q y+h(p, q)$ for arbitrary $h$. Setting $h=0$, we get the Legendre transformation [10]:

$$
X=p, \quad Y=q, \quad Z=-z+p x+q y, \quad P=x, \quad Q=y .
$$

This transformation maps (62) to

$$
\mathscr{A} Z=a(X, Y) T-2 b(X, Y) S+c(X, Y) R=0,
$$

with $T=Z_{Y Y}, S=Z_{X Y}$ and $R=Z_{X X}$.

3.4. Lie's theorem on the Monge-Ampère equation. We consider the MongeAmpère equation

$$
f=A\left(r t-s^{2}\right)+B r+C s+D t+E=0,
$$

where $A, B, C, D$ and $E$ are functions of $\omega^{(1)}$. In the study of this equation, the concept of intermediate integrals plays an important role [11]. An equation

$$
I\left(\alpha\left(\omega^{(1)}\right), \beta\left(\omega^{(1)}\right)\right)=0
$$

$\alpha: w^{(1)} \rightarrow R, \beta: w^{(1)} \rightarrow R, I(\alpha, \beta)$ an arbitrary function $R^{2} \rightarrow R$, is said to be a general intermediate integral of (72) if $\alpha$ and $\beta$ satisfy the equality

$$
f \equiv\left(D_{x} \alpha\right)\left(D_{y} \beta\right)-\left(D_{y} \alpha\right)\left(D_{x} \beta\right) .
$$

Lie [12] proved a theorem which in our notation reads as:

THEOREM (LIE). A Monge-Ampère equation admitting two general intermediate integrals $I^{1}\left(\alpha^{1}, \beta^{1}\right)=0$ and $I^{2}\left(\alpha^{2}, \beta^{2}\right)=0$ is transformable to the equation $Z_{X Y}=0$ by a Lie contact transformation $\Omega^{(1)}=\bar{\Omega}^{(1)}\left(\omega^{(1)}\right)$. Four of the components of $\bar{\Omega}^{(1)}$ are given by

$$
\bar{X}=\alpha^{1}, \quad \bar{Y}=\alpha^{2}, \quad \bar{P}=\beta^{1}, \quad \bar{Q}=\beta^{2} .
$$

We now show that these intermediate integrals are related to an invariance group:

Proposition 6. A Monge-Ampère equation possessing two general intermediate integrals $I^{1}\left(\alpha^{1}, \beta^{1}\right)=0$ and $I^{2}\left(\alpha^{2}, \beta^{2}\right)=0$ admits generators

$$
l_{i}=\sigma\left(\omega^{(1)}\right) I^{i}\left(\alpha^{i}, \beta^{i}\right) \partial_{z}, \quad i=1,2,
$$

$\sigma^{-1}=\rho=\left[\alpha^{1}, \beta^{1}\right]=\left[\alpha^{2}, \beta^{2}\right]$. 
Proof. It is easy to check that the equation $Z_{X Y}=0$ admits generators $L_{1}=$ $I^{1}(X, P) \partial_{Z}$ and $L_{2}=I^{2}(Y, Q) \partial_{Z}$ with arbitrary functions $I^{1}$ and $I^{2}$. On the other hand, according to Lie's theorem, there exists a Lie contact transformation $\Omega^{(1)}=\bar{\Omega}^{(1)}\left(\omega^{(1)}\right)$ mapping the Monge-Ampère equation in question to $Z_{X Y}=0$. In view of (75) and Lemma 2, the inverse of this transformation maps $L_{1}$ and $L_{2}$ to (76).

Now we are able to relate Theorem 1 and Lie's theorem. According to Theorem 1 , any linearizable Monge-Ampère equation admits a generator of the form (27). To see this we choose special forms for $I^{i}$, namely $I^{1}=I^{1}\left(\alpha^{1}\right)$ and $I^{2}=I^{2}\left(\alpha^{2}\right)$, let $l=l_{1}+l_{2}$, and then obtain a generator of the form

$$
l=\sigma \cdot\left(I^{1}\left(\alpha^{1}\right)+I^{2}\left(\alpha^{2}\right)\right) \partial_{z} \equiv \sigma \cdot U\left(\alpha^{1}, \alpha^{2}\right) \partial_{z} .
$$

Observing that $U(X, Y)=I^{1}(X)+I^{2}(Y)$ is the general solution of the differential equation $U_{X Y}=0$, we see that indeed these Monge-Ampère equations admit a generator of the form (27) with $\bar{X}=\alpha^{1}\left(\omega^{(1)}\right)$ and $\bar{Y}=\alpha^{2}\left(\omega^{(1)}\right)$.

These observations show that we can always find Lie's linearization of a MongeAmpère equation to $Z_{X Y}=0$, when it exists, by examining the invariance group of the equation and applying Theorem 1 . form

3.5. The equation $\left(z_{x}\right)^{\alpha} z_{x x}-z_{y y}=0$. A special Monge-Ampère equation of the

$$
c\left(z_{x}\right) z_{x x}-z_{y y}=0
$$

arises in a variety of physical problems such as nonlinear vibrations [13] $\left(c\left(z_{x}\right)>0\right)$, and irrotational transonic flows [14] $\left(c\left(z_{x}\right)=1+a z_{x}\right)$. In the following, we consider a class of equations of the form

$$
f=\left(z_{x}\right)^{\alpha} z_{x x}-z_{y y}=0, \quad \alpha \text { real, }
$$

and apply the foregoing analysis to examine a possible mapping to a linear equation.

The invariance group of (79) depends upon the value of $\alpha$. Assuming a generator to be of the form $l=\theta\left(\omega^{(1)}\right) \partial_{z}$, we find the following cases:

1) $\alpha \neq 0,-2,-4$ :

$$
\begin{gathered}
l_{1}=\left\{-(\alpha+4) x p q+\alpha z q-2(\alpha+1) y q^{2}-4 y \int p^{\alpha+1} d p\right\} \partial_{z}, \\
l_{2}=\{(\alpha+4) x p+(3 \alpha+4) y q-\alpha z\} \partial_{z}, \\
l_{3}=\left(z+\frac{1}{2} \alpha y q\right) \partial_{z}, \quad l_{4}=y \partial_{z}, \quad l_{5}=U(p, q) \partial_{z} .
\end{gathered}
$$

2) $\alpha=-2$ :

$$
l_{1}, l_{2}, l_{3}, l_{4}, l_{5} \text { as above, } \quad l_{6}=z p \partial_{z} \text {. }
$$

3) $\alpha=-4$ :

$$
\begin{aligned}
& l_{1}=(x p-y q) \partial_{z}, \quad l_{2}=U(p, q) \partial_{z}, \\
& l_{3}=\left\{p I^{1}\left(p^{-1}-q,\left(p^{-1}-q\right) y+z\right)\right\} \partial_{z}, \\
& l_{4}=\left\{p I^{2}\left(p^{-1}+q,\left(p^{-1}+q\right) y-z\right)\right\} \partial_{z} .
\end{aligned}
$$

4) $\alpha=0$ :

$$
\begin{gathered}
l_{1}=z \partial_{z}, \quad l_{2}=h(x, y) \partial_{z}, \\
l_{3}=I^{1}(x+y, p+q) \partial_{z}, \quad l_{4}=I^{2}(x-y, p-q) \partial_{z} .
\end{gathered}
$$


In these expressions $U(p, q)$ represents an arbitrary solution of the differential equation

$$
U_{p p}-p^{\alpha} U_{q q}=0
$$

and $I^{1}$ and $I^{2}$ are arbitrary functions of their arguments. In the last case $h(x, y)$ is an arbitrary solution of the differential equation $h_{x x}-h_{y y}=0$.

In all these cases, except for $\alpha=0$, the equation admits $l=U(p, q) \partial_{z}$ which is related to the above Legendre transformation.

The case $\alpha=-4$ is somewhat special. It is easy to verify that $I^{i}=0, i=1,2$, are the general intermediate integrals of the equation. As was previously discussed, this Monge-Ampère equation admits the generator (77), i.e.,

$$
l=\left\{p U\left(p^{-1}-q, p^{-1}+q\right)\right\} \partial_{z}
$$

for any $U(X, Y)$ satisfying the differential equation $U_{X Y}=0$. We now apply Theorem 1. Comparing (85) with (27), we have $\bar{X}=p^{-1}-q, \bar{Y}=p^{-1}+q, \sigma=p$. Using $[\bar{X}, \bar{Z}]=$ $[\bar{Y}, \bar{Z}]=0,(12)$, and $(30)$, we find the complete Lie contact transformation to be

$$
\begin{array}{cc}
X=p^{-1}-q, \quad Y=p^{-1}+q, & Z=-2 p^{-1}(z-p x-q y), \\
P=-\left(p^{-1}-q\right) y-z, & Q=\left(p^{-1}+q\right) y-z .
\end{array}
$$

The inverse transformation is

$$
\begin{gathered}
x=\frac{1}{2} Z-\frac{1}{4}(X+Y)(P+Q), \quad y=-(X+Y)^{-1}(P-Q), \\
z=-(X+Y)^{-1}(Y P+X Q), \quad p=2(X+Y)^{-1}, \quad q=\frac{1}{2}(-X+Y) .
\end{gathered}
$$

The transformation (86) maps the equation $\left(z_{x}\right)^{-4} z_{x x}-z_{y y}=0$ to the equation $Z_{X Y}=0$. If we introduce the general solution of $U_{X Y}=0$, i.e.,

$$
U(X, Y)=F(X)+G(Y), \quad F \text { and } G \text { arbitrary functions, }
$$

into (87) by $Z=U(X, Y)$, then we obtain a parametric representation of the general solution of the differential equation

$$
\left(u_{x}\right)^{-4} u_{x x}-u_{y y}=0
$$

Explicitly this is

$$
\begin{aligned}
& x=\frac{1}{2}(F(X)+G(Y))-\frac{1}{4}(X+Y)\left(F^{\prime}(X)+G^{\prime}(Y)\right), \\
& y=-(X+Y)^{-1}\left(F^{\prime}(X)-G^{\prime}(Y)\right), \\
& u=-(X+Y)^{-1}\left(Y F^{\prime}(X)+X G^{\prime}(Y)\right),
\end{aligned}
$$

where $F^{\prime}$ and $G^{\prime}$ denote derivatives.

We also see the following: for $\alpha \neq 0$, a differential equation of the form (84) can be mapped 1-1 into the equation $U_{X Y}=0$ if and only if $\alpha=-4$. The mapping is a composition of the Legendre transformation (70) relating $Z_{X X}-X^{-4} Z_{Y Y}=0$ to (79) and the transformation (86) relating (79) to $Z_{X Y}=0$. Explicitly, the transformation

$$
x=2(X+Y)^{-1}, \quad y=\frac{1}{2}(-X+Y), \quad z=(X+Y)^{-1} Z
$$

maps the equation $z_{x x}-x^{-4} z_{y y}=0$ to the equation $Z_{X Y}=0$.

4. Remarks on noninvertible mappings. In the preceding discussion we considered mappings transforming a nonlinear equation to a linear equation in a 1-1 manner. If we require only that a mapping transform a solution of some linear equation 
to a solution of a given nonlinear equation, the class of mappings widens and includes noninvertible (non-1-1) mappings. In the following we examine examples of such mappings and show that such mappings are often related to invariance under Lie groups.

\subsection{Burgers' equation. We consider Burgers' equation}

$$
\tilde{f}=\tilde{z}_{x x}+\tilde{z} \tilde{z}_{x}-\tilde{z}_{y}=0 \text {. }
$$

This equation admits a five-parameter point Lie group [15]. None of the generators is of the form (27), and hence by Theorem 1 there exists no 1-1 mapping to a linear equation. However it is known that a non-1-1 mapping, namely the Hopf-Cole transformation [16], [21]

$$
x=X, \quad y=Y, \quad \tilde{z}=\frac{2 Z_{X}}{Z},
$$

relates $(90)$ to the heat equation.

Introducing the transformation (91) into (90), we find that

$$
\tilde{f}=2 Z^{-2}\left(Z Z_{X X X}-Z_{X} Z_{X X}-Z Z_{X Y}+Z_{X} Z_{Y}\right)=0
$$

which factorizes as

$$
\tilde{f}=2 Z^{-2}\left(Z D_{X}-Z_{X}\right)\left(Z_{X X}-Z_{Y}\right)=0 .
$$

It follows from (93) that the transformation (91) maps a solution of the heat equation $Z_{X X}-Z_{Y}=0$ to a solution of Burgers' equation. It is incorrect to say that the Hopf-Cole transformation maps Burgers' equation to a linear equation. Although this type of mapping is out of the scope of the preceding discussion, this particular transformation is found to be related to a Lie group.

One standard argument [17] to rationalize the Hopf-Cole transformation is to introduce $z$ through $\tilde{z}=z_{x}$ and, after integrating once, one considers

$$
z_{x x}+\frac{1}{2}\left(z_{x}\right)^{2}-z_{y}=0 \text {. }
$$

One then says, "by inspection", that the transformation

$$
x=X, \quad y=Y, \quad z=2 \ln c Z, \quad c \text { constant }
$$

maps (94) to the heat equation $Z_{X X}-Z_{Y}=0$ and from this follows the transformation (91). Equation (94) corresponds to the first example studied in the previous section where we found the transformation (95) with $c=\frac{1}{2}$ by applying Theorem 1 .

4.2. The diffusion equation $D_{x}\left(\tilde{z}^{-2} \tilde{z}_{x}\right)-\tilde{z}_{y}=0$. We consider a nonlinear diffusion equation

$$
\tilde{f}=D_{x}\left(\tilde{z}^{-2} \tilde{z}_{x}\right)-\tilde{z}_{y}=0 .
$$

This equation admits a four-parameter point Lie group [4] with no generator of the form (27). As in the previous example we let $\tilde{z}=z_{x}$ and instead of equation (96) we consider an integrated form

$$
f=\left(z_{x}\right)^{-2} z_{x x}-z_{y}=0 .
$$

This equation admits [6] seven generators of point transformations including the generator

$$
l=U(z, y) z_{x} \partial_{z}
$$


involving an arbitrary solution of $U_{z z}-U_{y}=0$. Comparing this with (27), we find a point transformation

$$
X=z, \quad Y=y, \quad Z=x
$$

which maps (97) to the heat equation $\mathscr{A} Z=Z_{X X}-Z_{Y}=0$. From (99) it follows that $\tilde{z}=z_{x}=\left(Z_{X}\right)^{-1}$ and one can verify that the transformation

$$
x=Z, \quad y=Y, \quad \tilde{z}=\left(Z_{X}\right)^{-1}
$$

transforms $(96)$ to

$$
\tilde{f}=Z_{X}^{-3}\left(Z_{X} D_{X}-Z_{X X}\right)\left(Z_{X X}-Z_{Y}\right)=0 .
$$

Hence the transformation (100) maps a solution of the heat equation to a solution of (96).

The transformation (100) was previously found by separate analyses [18], [19].

The mapping in the following example cannot be related to Theorem 1.

4.3. The Liouville equation $z_{x y}-e^{z}=0$. We consider the Liouville equation

$$
z_{x y}-e^{z}=0
$$

This equation admits a generator

$$
l=\left[f(x) z_{x}+g(y) z_{y}+f_{x}(x)+g_{y}(y)\right] \partial_{z}
$$

with two arbitrary functions $\{f(x), g(y)\}$ and their derivatives. It does not admit a generator of the form (27). The invariant solution $z=u(x, y)$ associated with the above generator (103) is defined as a solution of the system

$$
\begin{aligned}
& f(x) u_{x}+g(y) u_{y}+f_{x}(x)+g_{y}(y)=0, \\
& u_{x y}-e^{u}=0 .
\end{aligned}
$$

The solution is found to be

$$
u=\ln \left|\frac{2 \phi_{x} \psi_{y}}{(\phi+\psi)^{2}}\right|
$$

where $\phi(x)=\int f^{-1} d x$ and $\psi(y)=\int g^{-1} d y$. This is the general solution of the Liouville equation [11]. Introducing $U=\phi+\psi$, we write this as

$$
u=\ln \left|2 U^{-2} U_{x} U_{y}\right| \text {. }
$$

Recognizing $U$ as the general solution of the equation $U_{x y}=0$, we conclude that the transformation

$$
z=\ln \left|2 Z^{-2} Z_{x} Z_{y}\right|
$$

maps a solution of $Z_{x y}=0$ to a solution of $z_{x y}-e^{z}=0$. One can also see this from the equality

$$
z_{x} g y-e^{z}=\left(Z_{x}^{-1} D_{x}+Z_{y}^{-1} D_{y}-Z_{x}^{-2} Z_{x x}-Z_{y}^{-2} Z_{y y}-2 Z^{-1}\right) Z_{x y}
$$

hence $Z_{x y}=0 \rightarrow z_{x y}-e^{z}=0$.

5. Concluding remarks. In this work we have shown that by examining the invariance group of a system of nonlinear differential equations we can determine definitively whether the equation is transformable to a linear system by a 1-1 mapping. Moreover the mapping can be constructed from a generator of the group. In all cases, 
we need only consider group generators of the form (49) or $\left(49^{\prime}\right)$ depending on no derivative higher than those of first order.

The question of the existence of noninvertible mappings relating linear and nonlinear equations is more complex. The problem of finding such a mapping is equivalent to finding a condition under which a given nonlinear equation admits a transformation leading to a factorization such as (93), (101) and (109). It was pointed out previously [19] that the existence of an infinite sequence of Lie-Bäcklund invariance groups for a given equation indicates the possibility of such a transformation, and this was used to construct the transformation (100). Such a sequence also exists for Burgers' equation [20].

Finally, with our approach it should be emphasized that even if one is unable to linearize given nonlinear differential equations, one is always left with their invariance groups. In turn these can be used for the construction of invariant solutions, conservation laws and other invariance properties of the equations.

\section{REFERENCES}

[1] G. W. Bluman, in Symmetry, Similarity and Group Theoretic Methods in Mechanics, P. G. Glockner and M. C. Singh, eds., Univ. of Calgary Press, Calgary, 1974.

[2] - On the transformation of a diffusion process to the Wiener process, this Journal, 39 (1980), pp. 238-247.

[3] S. LiE, Gesammelte Abhandlungen, vols 3-4. Teubner, Leipzig, 1922; Reprints, Johnson Reprint Corp., New York, 1973.

[4] L. V. Ovsuannikov, Group Properties of Differential Equations, G. W. Bluman, trans., Siberian Section of the Academy of Science of U.S.S.R., Novosibirsk, 1962.

[5] — Group Analysis of Differential Equations, Nauka, Moscow, 1978. (In Russian.)

[6] G. W. Bluman ANd J. D. Cole, Similarity Methods for Differential Equations, Springer-Verlag, New York, 1974.

[7] A. V. BÄCKLUND, Über Flachenstransformationen, Math. Ann., 9 (1876), pp. 297-320.

[8] E. A. Müller AND K. Matschat, Über das Auffinden von Ähnlichkeitslösungen partieller Differentialgleichungssysteme unter Benutzung von Transformationsgruppen, mit Anwendungen auf Probleme der Strömungsphysik, Miszellaneen der Angewandten Mechanik, Berlin, 1962, pp. $190 f$.

[9] A. R. Forsyth, Theory of Differential Equations, vol. 5, Cambridge University Press, Cambridge, 1906.

[10] R. Courant ANd D. Hilbert, Methods of Mathematical Physics, vol. 2, Interscience, New York, 1962.

[11] A. R. Forsyth, Theory of Differential Equations, vol. 6, Cambridge University Press, Cambridge, 1906.

[12] S. LIE, Neue Integrationsmethode der Monge-Ampèreshen Gleichung, Arch. Math. Kristiania, 2 (1877), pp. 1-9; also in [11].

[13] N. J. ZABUSKY, Exact solutions for the vibrations of nonlinear continuous model strings, J. Math. Phys., 3 (1962), pp. 1028-1039.

[14] C. Ferrari and F. G. Tricomi, Transonic Aerodynamics, Academic Press, New York, 1968.

[15] V. L. Katkov, Group classification of solutions of the Hopf equation, Ž. Prikl. Meh. i Tehn. Fiz., 6 (1965), pp. 105ff. (In Russian.)

[16] E. HOPF, The partial differential equation $u_{t}+u u_{x}=u_{x x}$, Comm. Pure Appl. Math., 3 (1950), pp. 201-230.

[17] G. B. Whitham, Linear and Nonlinear Waves, Wiley-Interscience, New York, 1974.

[18] G. Rosen, Nonlinear heat conduction in solid $\mathrm{H}_{2}$, Phys. Rev. B, 19 (1979), pp. 2398-2399.

[19] G. W. BlumAN AND S. KUMEI, On the remarkable nonlinear diffusion equation $(\partial / \partial x)\left[a(u+b)^{-2} \times\right.$ $(\partial u / \partial x)]-\partial u / \partial t=0$, J. Math. Phys., 21 (1980), pp. 1019-1023.

[20] P. OLVER, Evolution equations possessing infinitely many symmetries, J. Math. Phys., 18 (1977), pp. 1212-1215.

[21] J. D. COLE, On a quasilinear parabolic equation occurring in aerodynamics, Quart. Appl. Math., 9 (1951), pp. 225-236. 